\title{
COVID-19-Induced Psychosis and Suicidal Behavior: Case Report
}

\author{
Mason Chacko ${ }^{1,2} \cdot$ Asha Job $^{1} \cdot$ Fred Caston III ${ }^{1} \cdot$ Prem George $^{3} \cdot$ Adeeb Yacoub $^{1} \cdot$ Ricardo Cáceda $^{1,4}$ \\ Accepted: 17 September 2020 / Published online: 26 September 2020 \\ (C) This is a U.S. Government work and not under copyright protection in the US; foreign copyright protection may apply 2020
}

\begin{abstract}
The COVID-19 pandemic is associated with different types of stressors: fear of infection, financial burden, and social isolation. Additionally, COVID-19 infection seems to increase the risk for neuropsychiatric symptoms including psychosis. We present a case of a 52-year-old male with no previous psychiatric history who developed severe paranoia leading to a suicide attempt. He was successfully treated with a combination of milieu treatment, pharmacotherapy, and electroconvulsive therapy. We add to the nascent literature that COVID-19, as other coronaviruses, can increase the risk for severe psychosis and suicidal behavior.
\end{abstract}

Keywords COVID-19 $\cdot$ Suicide $\cdot$ Psychosis · Depression

\section{Introduction}

The emergence and global spread of the 2019 novel coronavirus (COVID-19) has imposed a significant mental health burden on communities around the world. Many of the psychiatric manifestations of COVID-19 are a consequence of psychological stressors, such as fear of illness and death, prolonged social isolation, and uncertainty and fear about the future. However, a growing body of evidence suggests that the virus itself can precipitate psychosis among infected individuals [1]. Emerging neuropsychiatric sequelae of COVID-19 include encephalopathy, anxiety, depression, mania, and trauma-related disorders [2]. The potential for the virus to induce psychosis is of particular interest given the association with acute suicidal/homicidal ideation and other severe perceptual and behavioral disturbances. Much is yet to be discovered about the biological mechanisms, presentation, treatment,

This article is part of the Topical Collection on Covid-19

Mason Chacko

mason.chacko@stonybrookmedicine.edu

1 Department of Psychiatry and Behavioral Health, Stony Brook University, Stony Brook, NY, USA

2 Department of Hospital Medicine, Southside Hospital, Bay Shore, NY, USA

3 OP Private Practice/SUNY Downstate, Brooklyn, NY, USA

4 Psychiatry Service, Northport VA Medical Center, Northport, NY, USA and long-term outcomes of COVID-related psychotic illness.

A connection with psychosis has been described in other strains of coronavirus, including the species responsible for severe acute respiratory syndrome (SARS-CoV). Common symptoms observed in SARS-related psychosis include persecutory delusions, auditory hallucinations of gossip, suicidal thoughts and behavior, and sleep disturbances [3]. One survey of recently discharged SARS patients found that disease severity - as measured by chest radiograph on admission, maximum oxygen requirement, need for ICU care, and length of hospital stay-was an independent predictor of new onset psychosis and behavioral problems in both the acute and convalescent stages of illness [4]. Compared with SARS patients without psychiatric complications, SARS patients with new onset psychosis had higher rates of family history of psychiatric illness, as well as higher rates of concurrent SARS infection in family members $[3,5]$. This data suggests that personal vulnerability and psychosocial stressors may contribute, in part, to the psychosis associated with coronavirus infection. Furthermore, these findings argue against the etiological theory that SARS-related psychosis is simply a result of corticosteroid therapy. Another study found that patients with new onset psychosis have a significantly higher seroprevalence of IgG antibodies against four human coronavirus strains (229E, HKU1, NL63, and OC43) compared with non-psychiatric controls [6]. These findings suggest that coronavirus exposure may be a risk factor for development of psychosis in susceptible individuals. 
Psychosis caused by viral infection has been associated with other novel infectious disease outbreaks. One study reviewing literature from the H1N1 influenza, Ebola, SARS, Middle East Respiratory Syndrome (MERS), and COVID-19 outbreaks estimated that 0.9 to $4 \%$ of people exposed to a virus during an epi- or pandemic develop psychosis or psychotic symptoms, significantly higher than the median incidence rate of $.015 \%$ for psychosis in the general population [7]. Researchers also found that low doses of atypical antipsychotics, particularly aripiprazole, are effective in treating psychosis associated with viral infection. Several case reports have recently been published describing patients with no psychiatric history who developed acute psychosis in the setting of positive diagnostic testing for COVID-19 [8]. Common themes observed in these patients include severe anxiety, agitation, suspiciousness, and auditory hallucinations. None of the patients displayed any of the typical respiratory or constitutional symptoms associated with COVID-19 but they did show signs of systemic inflammation as evidenced by elevated C-reactive protein (CRP) and other inflammatory markers.

Here, we report the case of a patient who presented with disorganized behavior and paranoia leading to a suicide attempt in the context of positive serological testing for COVID-19 antibodies (IgG).

\section{Case Presentation}

Mr. C, a 52-year-old male with medical history of obstructive sleep apnea on home CPAP with no significant psychiatric history, presented to our emergency department (ED) with acute altered mental status and mutism. As per family account Mr. C had been "off" for 1 week, with decreased speech and paranoid delusions that he was the cause of the "coronavirus pandemic." The ED visit was precipitated by the onset of mutism. His vitals were $99.2^{\circ} \mathrm{F}$, blood pressure $181 / 125$, pulse 120 , and respiratory rate 20 . Based upon his initial presentation and vitals, a code stroke was activated. A noncontrast head computerized tomography (CT) and magnetic resonance imaging with diffusion weight imaging was negative for acute pathology. However, his blood work was significant for elevated liver transaminases along with acute inflammatory markers (erythrocyte sedimentation rate $=40, \mathrm{C}$ reactive protein $=1.5$, and D-dimer $=1003$ ). His COVID-19 PCR was negative. His urine analysis was indicative of dehydration, with positive ketone and high specific gravity. In the setting of elevated D-dimer, the patient underwent a chest CT scan to rule out pulmonary embolism, which was negative; however, he was found to have multifocal pneumonia. Mr. C received intravenous antibiotics including ceftriaxone and azithromycin along with lorazepam $2 \mathrm{mg}$ IV push $\times 2$ doses. He was subsequently admitted to the observational unit for psychiatry consult. At that time, his psychotic and catatonic symptoms appeared to have resolved. He reported that two of his colleagues with whom he rides had tested positive for COVID-19. This led to him becoming anxious and feeling overwhelmed. There were no features of catatonia. He was subsequently discharged home.

Mr. C returned 2 days later to the psychiatric emergency room complaining of worsening agitation, anxiety, paranoia, and mutism. He received haloperidol $5 \mathrm{mg}$ IM and lorazepam $2 \mathrm{mg}$ IM prior to formal evaluation. He was found to be disorganized, irritable, and with select mutism. As per history from the family, he awoke that morning stating: "Today I should die!" and attempted to cut his neck with a knife. Mr. $\mathrm{C}$ was subsequently admitted to the inpatient psychiatric ward and was started on fluoxetine $20 \mathrm{mg}$ po once daily, olanzapine $5 \mathrm{mg}$ po BID, and lorazepam $1 \mathrm{mg}$ po BID. In the inpatient psychiatric unit, $\mathrm{Mr}$. $\mathrm{C}$ was found to be isolated, irritable, and attempted to abscond. He remained tachycardic and Medicine was consulted and recommended that he be transferred to the Medicine service for re-testing of COVID-19. He had a subsequent negative PCR for COVID-19 (second test) and psychiatric CL service was consulted. His lorazepam was increased to $2 \mathrm{mg}$ po BID but he remained irritable and attempted to cut his wrist with a plastic knife from his breakfast tray. After he was cleared by Medicine, he was transferred back to the inpatient psychiatric ward. Mr. C remained paranoid but was agreeable to electroconvulsive therapy (ECT). He underwent a total of 6 ECT treatments which led to his overall clinical improvement with no further thoughts of suicide. His discharge medications included olanzapine $2.5 \mathrm{mg}$ oral QHS, sertraline $100 \mathrm{mg}$ po once daily, clonazepam $0.25 \mathrm{mg}$ BID, and trazadone $50 \mathrm{mg}$ po qhs. Mr. C underwent IgG antibody testing for COVID-19 3 weeks after discharge and was found positive. He continued follow-up on the outpatient psychiatric and remained symptom-free as of 4 weeks post-discharge.

From the patient's perspective, it was a very confusing experience. Several weeks after hospital discharge, he was unable to recall specific details or strong emotions related to hospitalization.

\section{Discussion}

This case shows the potential for a coronavirus-associated inflammatory trigger to precipitate psychosis in seropositive patients. Although it is possible that excessive fear and anxiety related to the pandemic may have contributed to this patient's psychotic symptoms, the role of viral exposure in the development of his psychosis must be explored. The connection between COVID-19 and new onset psychosis was first observed in China, the country with the earliest documented cases of the virus. A retrospective chart review in the city of Xuzhou showed a $10 \%$ increase in psychosis among first time 
psychiatric outpatients in January 2020 compared with averaged data from January 2017-2019 [9]. There was also a 25\% rise in psychosis in late January 2020 versus early January 2020 , with linear regression analysis revealing a strong positive relationship between psychosis incidence and number of confirmed COVID-19 cases. The study also found that mean age of first episode psychosis increased significantly from 39 (2017-2019) to 50 (2020), suggesting that age is a major risk factor for COVID-related new onset psychosis. Our patient, a 52-year-old male, is illustrative of the demographic changes highlighted in the Xuzhou study. The fact that elderly individuals have the highest risk of contracting and dying from the disease may explain the heightened psychological vulnerability of this population to the effects of the virus.

Recent reports of COVID-induced psychosis describe a clinical and laboratory presentation similar to that seen in our patient [8]. One case is of a 30-year-old male with no psychiatric history who presented with several days of severe anxiety, auditory hallucinations, psychogenic polydipsia, and suicidality. On exam, he was responding to internal stimuli and believed he was being followed. Psychosis resolved after 4 days of low-dose quetiapine. The second case describes a 34-year-old female with a history of panic disorder who was evaluated for paranoia, disorganized behavior, and perseverating on strange somatic sensations, such as migratory paresthesia and the feeling of a fire burning within her body. This patient required a more complex treatment regimen of benzodiazepines, antipsychotics, and antidepressants. The final case describes a 33-year-old male with opioid use disorder who presented with first episode psychosis, including intense agitation, persecutory delusions, and auditory hallucinations. His symptoms improved with low-dose quetiapine.

Many parallels can be drawn between these cases. In all three, the patients tested positive for COVID-19 but were physically asymptomatic with none of the respiratory, constitutional, or gastrointestinal symptoms typically seen with the disease. They also denied any history of anosmia or ageusia. All three patients were found to have elevated CRP, as well as elevated ferritin in the first patient. The pattern of psychotic symptoms seems to be similar across all cases, with most patients - including the one described in this reportpresenting with extreme anxiety, agitation, paranoia, auditory hallucinations, and suicidal ideation. Also like our patient, all three were fully alert and oriented on initial presentation despite their disorganized thought and behavior. This, in addition to the absence of any fluctuations in consciousness, makes the diagnosis of delirium less likely. Our patient also had elevated inflammatory markers on admission, including high CRP and ESR levels. Furthermore, our patient did not report any fever, chills, cough, sore throat, dyspnea, muscle aches, or fatigue - symptoms that are generally associated with COVID-19. Thus, it seems that patients who are prone to developing psychosis due to SARS-CoV-2 may be less vulnerable to the respiratory and systemic complications commonly caused by the virus.

First established in the late nineteenth century, the theory that microbial agents are etiologically linked to psychosis has received a new wave of scientific support in recent years [10]. One study found that patients with new onset psychosis had significantly higher serum and/or CSF IgG titers for at least one of the six viruses under investigation (cytomegalovirus, HSV1, mumps, measles, varicella zoster, Japanese encephalitis virus) compared with non-psychiatric controls. Many of the psychotic patients also had a diagnostic (four-fold) change in viral antibody titers over the 2-week study period, indicating active viral infection [11]. A number of case reports have been published describing persecutory delusions, poor self-care, vivid auditory and visual hallucinations, food refusal, and insomnia following infection with the mosquito-borne dengue virus and Zika virus [12-14]. Psychiatric complications associated with these two flaviviruses seem to follow a similar pattern with onset of psychosis occurring approximately 1 week after resolution of viral symptoms (e.g., fever, rash, myalgia) and excellent response to atypical antipsychotics. Untreated HIV infection has also been associated with new onset psychosis [15]. The risk of psychosis in these patients is shown to be positively correlated with HIV viral load and negatively correlated with CD4 count [16]. Symptoms in these patients generally improve with low-dose antipsychotics and initiation of antiretroviral therapy. Additional viral infections shown to be associated with acute psychosis include H1N1 influenza, Borna disease virus, and measles virus [17-19]. Many of the patients described in the cases above had no recall of their psychotic episode after recovery. Our patient, too, denied any recollection of his hospital course or the events surrounding his hospitalization. This sort of retrograde amnesia following resolution of psychosis has been described in many cases of brief psychotic disorder, with the patient losing all memory of the entire psychotic period [20].

Several pathological mechanisms have been proposed to explain the neuropsychiatric sequelae of COVID-19, including new onset psychosis. Given that neurotropism and neuroinvasive potential has been demonstrated in many strains of coronavirus, it is possible that direct viral infiltration into the central nervous system (CNS) is responsible for the neuropsychiatric manifestations of COVID-19 [1]. It is believed that coronaviruses can migrate from the respiratory tract to the brain via retrograde axonal transport from the olfactory bulb [21]. Another possible route involves hematogenous dissemination into the CNS via infected blood leukocytes. In mouse models, the human respiratory coronavirus strain OC43 (HCoV-OC43) is shown to have a preferential tropism for infecting neurons versus other neural cells (e.g., oligodendrocytes, astrocytes, microglia) [22]. Mice inoculated with HCoV-OC43 developed signs of acute encephalitis secondary to neuronal apoptosis. These mice were found to have 
behavioral abnormalities with persistence of viral RNA in the brain for several months. These findings suggest that respiratory viruses with neuroinvasive potential can induce neuronal cell death and consequent psychosis in vulnerable individuals.

Another proposed mechanism for COVID-related psychosis involves dysregulation of cytokine networks. Peripheral activation of proinflammatory cytokines (e.g., interleukin (IL)-6, tumor necrosis factor (TNF)-alpha, IL-8, IL-10, IL2R) in response to COVID-19 infection may contribute to neuroinflammation via increased permeability and compromised integrity of the blood-brain barrier [8]. Immunological triggers have long been shown to play a role in the pathogenesis of psychotic illness, including the psychosis associated with HIV infection and other viruses [23, 24]. All four of the patients with COVID-related psychosis discussed here presented with elevated CRP. This acute phase reactant has been implicated in the development of psychotic disorders, with $28 \%$ of schizophrenia patients showing high CRP levels [25]. Interestingly, the serotonin antagonist cinanserin, a drug originally developed to treat schizophrenia, has also proven effective in inhibiting replication of coronavirus strains SARS-CoV and HCoV-229E. This suggests a common neuropathological mechanism underlying both schizophrenia and the psychosis associated with coronavirus infection [26].

A third possible mechanism of psychosis in COVID-19 patients may be related to the severe sensory deprivation associated with hospital isolation measures. This can occur during at home quarantine, or subsequent isolation protocols in a single hospital room with minimal direct human contact. Similar cases of acute psychosis have been described in prisoners in solitary confinement and in experimental sensory deprivation. However, this does not seem to be the case with Mr. C, who was already psychotic when he presented to the hospital, and was previously living with his family.

The case of Mr. C demonstrates just one potential cause of increased suicide risk during the COVID-19 pandemic. Psychosis-associated suicidal behavior appears to be a rare, but severe neuropsychiatric sequelae of acute viral infection. However, several additional risk factors for suicide during the COVID-19 pandemic have been described recently. [27] Excessive apprehension about the spread and fatality of disease compounded by overexposure to media coverage can precipitate suicidal ideation and behavior in vulnerable individuals. Mass quarantine and physical distancing measures, while epidemiologically beneficial for slowing contagion, also carry severe psychological consequences that cannot be ignored. Moreover, growing stigma and social rejection of infected patients and their families may lead to guilt, anger, and alienation that further exacerbate psychological distress. Domestic violence, alcohol consumption, unemployment, and financial insecurity are additional risk factors for suicide that have risen in prevalence due to the COVID-19 pandemic. Finally, patients with mental illness and those who are acutely suicidal may have reduced access to the resources and care they need. Successful suicide prevention during the pandemic requires targeted interdisciplinary interventions in order to mitigate the impact of these multiple risk factors.

\section{Conclusion}

New onset psychosis is a not so common described symptom of COVID-19 infection with potential lethal consequences, which needs aggressive and early management in order to decrease morbidity and mortality.

\section{Compliance with Ethical Standards}

Conflict of Interest The authors declare that they have no conflict of interest.

Ethical Approval At Stony Brook University, a single case report does not require IRB review and approval since it does not meet their definition of research. Additional information can be found on the Stony Brook University IRB website in their Statement of Purpose, or provided if requested.

Informed Consent The patient signed a consent authorizing the writing and publication of this case report.

\section{References}

1. Troyer EA, Kohn JN, Hong S. Are we facing a crashing wave of neuropsychiatric sequelae of COVID-19? Neuropsychiatric symptoms and potential immunologic mechanisms. Brain Behav Immun [Epub ahead of print]. 2020;87:34-9.

2. Rogers JP, Chesney E, Oliver D, et al. (2020). Psychiatric and neuropsychiatric presentations associated with severe coronavirus infections: a systematic review and meta-analysis with comparison to the COVID-19 pandemic. Lancet Psychiatry [Epub ahead of print].

3. Cheng SKW, Tsang JSK, Ku KH, Wong CW, Ng YK. Psychiatric complications in patients with severe acute respiratory syndrome (SARS) during the acute treatment phase: a series of 10 cases. $\mathrm{Br} \mathrm{J}$ Psychiatry. 2004;184:359-60.

4. Sheng B, Cheng SKW, Lau KK, et al. The effects of disease severity, use of corticosteroids and social factors on neuropsychiatric complaints in severe acute respiratory syndrome (SARS) patients at acute and convalescent phases. Euro Psychiat. 2005;20(3):23642.

5. Lee DTS, Wing YK, Leung HCM, Sung JJY, Ng YK, Yiu GC, et al. Factors associated with psychosis among patients with severe acute respiratory syndrome: a case-control study. Clin Infect Dis. 2004;39(8):1247-9.

6. Severance EG, Dickerson FB, Viscidi RP, Bossis I, Stallings CR, Origoni AE, et al. Coronavirus immunoreactivity in individuals with a recent onset of psychotic symptoms. Schizophr Bull. 2011;37(1):101-7.

7. Brown E, Gray R, Monaco SL, et al. The potential impact of COVID-19 on psychosis: a rapid review of contemporary epidemic and pandemic research. Schizophr Res. 2020. 
8. Ferrando SJ, Klepacz L, Lynch S, et al. (2020). COVID-19 psychosis: a potential new neuropsychiatric condition triggered by novel coronavirus infection and the inflammatory response? Psychosomatics [Epub ahead of print].

9. $\mathrm{Hu} \mathrm{W}, \mathrm{Su} \mathrm{L}$, Qiao J, et al. (2020). COVID-19 outbreak increased risk of schizophrenia in aged adults. PsyChinaXiv.

10. Yolken RH, Torrey EF. Are some cases of psychosis caused by microbial agents? A review of the evidence. Mol Psychiatry. 2008;13(5):470-9.

11. Srikanth S, Ravi V, Poornima KS, Shetty KT, Gangadhar BN, Janakiramaiah N. Viral antibodies in recent onset, nonorganic psychoses: correspondence with symptomatic severity. Biol Psychiatry. 1994;36(8):517-21.

12. Chavez ME, Rojas M, Fortea A. Post dengue psychosis: report of 4 cases. Eur Neuropsychopharmacol. 2016;26:S574-5.

13. Chaudhury S, Jagtap B, Ghosh D. Psychosis in dengue fever. Medic J Dr DY Patil Univ. 2017;10(2):202-4.

14. Corrêa-Oliveira GE, Amaral JL, Fonseca BA, et al. Zika virus infection followed by a first episode of psychosis: another flavivirus leading to pure psychiatric symptomatology. Braz J Psychiat. 2017;39(4):381-2.

15. Carter AT, Bulbena A, Dunn N, et al. New-onset psychosis in patient with untreated HIV. Euro Psychiat. 2016;33:S655.

16. Laher A, Ariefdien N, Etlouba Y. HIV prevalence among firstpresentation psychotic patients. HIV Med. 2018;19(4):271-9.

17. Teng JY, Lee TS. Persistent mania and psychosis in a case of novel influenza a (H1N1) virus encephalitis. Euro Psychiat. 2011;26(1): 406.

18. Zaliunaite V, Steibliene V, Bode L, Podlipskyte A, Bunevicius R, Ludwig H. Primary psychosis and Borna disease virus infection in Lithuania: a case control study. BMC Psychiat. 2016;16(1):369.
19. Dickerson F, Stallings C, Origoni A, et al. Antibodies to measles in individuals with recent onset psychosis. Schizophr Res. 2010;119(1):89-94.

20. Stevens J. Brief psychoses: do they contribute to the good prognosis and equal prevalence of schizophrenia in developing countries? Br J Psychiatry. 1987;151(3):393-6.

21. Desforges M, Coupanec AL, Dubeau P, et al. Human coronaviruses and other respiratory viruses: underestimated opportunistic pathogens of the central nervous system? Viruses. 2019;12(1):1-28.

22. Jacomy H, Fragoso G, Almazan G, Mushynski WE, Talbot PJ. Human coronavirus OC43 infection induces chronic encephalitis leading to disabilities in BALB/C mice. Virology. 2006;349(2): $335-46$.

23. Upthegrove R, Khandaker GM. Cytokines, oxidative stress and cellular markers of inflammation in schizophrenia. Neuroinflam Schizophrenia Curr Topics Behav Neurosci. 2020;44:49-66.

24. Munjal S, Ferrando SJ, Freyberg Z. Neuropsychiatric aspects of infectious diseases: an update. Crit Care Clin. 2017;33(3):681-712.

25. Miller BJ, Culpepper N, Rapaport MH. C-reactive protein levels in schizophrenia. Clin Schizophrenia Related Psychos. 2014;7(4): 223-30.

26. Chen L, Gui C, Luo X, Yang Q, Günther S, Scandella E, et al. Cinanserin is an inhibitor of the $3 \mathrm{C}$-like proteinase of severe acute respiratory syndrome coronavirus and strongly reduces virus replication in vitro. J Virol. 2005;79(11):7095-103.

27. Gunnell D, Appleby L, Arensman E, Hawton K, John A, Kapur N, et al. Suicide risk and prevention during the COVID-19 pandemic. Lancet Psychiatry. 2020;7(6):468-71.

Publisher's Note Springer Nature remains neutral with regard to jurisdictional claims in published maps and institutional affiliations. 